\title{
IMAGE DENOISING USING ADAPTIVE SUBBAND DECOMPOSITION
}

\author{
Sinan Gezici ${ }^{1}$, Ismail Yilmaz ${ }^{1}$, Omer N. Gerek ${ }^{2}$, A. Enis Çetin ${ }^{1}$ \\ ${ }^{1}$ Department of Electrical and Electronics Engineering, \\ Bilkent University, Ankara, Turkey \\ ${ }^{2}$ Anadolu University, Eskisehir, Turkey \\ E-mail: cetin@ee.bilkent.edu.tr
}

\begin{abstract}
In this paper, we present a new image denoising method based on adaptive subband decomposition (or adaptive wavelet transform) in which the filter coefficients are updated according to an Least Mean Square (LMS) type algorithm. Adaptive subband decomposition filter banks have the perfect reconstruction property. Since the adaptive filterbank adjusts itself to the changing input environments denoising is more effective compared to fixed filterbanks. Simulation examples are presented.
\end{abstract}

\section{INTRODUCTION}

In this paper, a new image denoising method based on adaptive subband decomposition $[1,2,3]$ is presented. In most denoising applications a fixed wavelet transform is used to process the entire image $[4,5]$. However, a typical image consists of regions with different characteristics. The main idea of this paper is to use space varying filterbanks instead of fixed wavelets to process the image.

The concept of the adaptive filterbanks are introduced in $[6,7,8]$. Classical adaptive prediction concepts are combined with the Perfect Reconstruction Filter Banks (PRFB) in $[1,2]$ where the key idea is to decorrelate the polyphase components of the multichannel structure by using an adaptive predictor. In $[1,2,3]$ these structures are used for image coding in which the filters adapt to the changing input conditions according to an adaptation strategy such as the Least Mean Square (LMS) algorithm. Since adaptive filterbanks adapt to the changing input environments denoising is more effective compared to the regular fixed filterbanks. Our adaptive filterbanks are based on polyphase structures and they have the perfect reconstruction property. In our approach, the upperbranch is the lowpass filtered and downsampled version of the original signal, and the lower branch signal is basically the adaptive prediction error. In the two channel filterbank the the samples of the lower branch is adaptively estimated using the samples of the lowpass fil- tered upper branch. Due to this reason, in image analysis the "low-high", "high-low" and "high-high" subimages correspond to unpredictable portion of the image data containing edges, noise etc. Therefore, in flat or slowly varying regions whatever appears in highbands essentially correspond to noise. Thresholding is a memoryless operation and it does not take into account the correlation among neighboring coefficients. Therefore the data should be correlated as much as possible before thresholding. In this sense adaptive subband decomposition is suitable for image denoising as it tries to decorrelate not only the channels but also the neighboring image samples.

In the next section, we introduce two adaptive filterbanks that we use in image denoising. In Section 3, we discuss several denoising strategies, and in Section 4, we present simulation examples.

\section{ADAPTIVE FILTER BANKS}

The concepts of adaptive filtering and subband decomposition have been previously used together by a number of researchers [10]-[13]. Most of the proposed adaptation algorithms for subband decomposition filter banks consider the problem of system identification and noise removal [10][13]. In these works, the adaptive filtering problem is considered in the subband domain. The issues of efficient complex or real valued filter design methods to increase subband domain adaptive filtering performance is also investigated in [10] in which the design of the filter bank satisfying the prespecified requirements for adaptive filtering in subbands is studied.

The choice of subband filter banks according to the input signal is also considered by some researchers [14]-[15]. The main goal of these works is to find the best wavelet basis for decomposing the entire data, and fixed filter banks chosen according to an optimality criterion are used throughout the entire duration or extent of the signal whereas in this paper the filters vary as the nature of the input changes. 
Our adaptation scheme is different from the subband adaptive filter structures which performs adaptive filtering in the subbands [10]- [13]. The adaptation scheme in our method neither tries to estimate an unknown system nor uses a fixed filter bank throughout the entire duration of the signal. Our scheme inherently updates the filter banks and finds ideal filters for each signal sample while preserving the perfect reconstruction property.

In Figure $1, x_{1}(n)$ is the downsampled version of the original signal, $x(n)$, thus it consists of the even samples of $x(n)$. Similarly, the signal $x_{2}(n)$ consists of the odd samples. An LMS based FIR predictor of $x_{2}(n)$ from $x_{1}(n)$ can be expressed as

$$
\hat{x}_{2}(n)=\mathbf{w}(n) \mathbf{x}_{1}^{T}(n),
$$

where $\mathbf{x}_{1}(n)=\left[x_{1}(n-N), \ldots, x_{1}(n+M)\right]^{T}$ is the observation vector, and $\mathbf{w}(n)$ is the vector of predictor coefficients which is adapted by the equation

$$
\mathbf{w}(n+1)=\mathbf{w}(n)+\mu \frac{\mathbf{x}_{1}(n) e(n)}{\left\|\mathbf{x}_{1}(n)\right\|^{2}},
$$

where the error signal $e(n)$ is given by

$$
e(n)=x_{2}(n)-\hat{x}_{2}(n) .
$$

The PR property is preserved in this structure as long as the same adaptation algorithm is used at the analysis and the synthesis stages. Since the subsignal $x_{h}(n)$ as well as $x_{l}(n)$ are available both at the encoder and at the decoder, the synthesis stage can adapt the filter $P$ with the same filter tap coefficients $\hat{\mathbf{w}}(n)$ as long as the same adaptation strategy is used during the analysis and synthesis. Therefore, no side information needs to be transmitted for perfect reconstruction. This is an important property of the filterbank in image coding applications providing low bit rates. In image denoising applications this property is not that critical because adaptive filter coefficients can be stored and reused during reconstruction.

A weakness of the structure shown in Figure 1 is that the subsignal $x_{1}(n)$ may suffer from aliasing due to downsampling. Aliasing affects the quality of prediction especially when further decompositions over $x_{1}(n)$ are carried out. In order to eliminate this problem an anti-aliasing filtering stage is introduced in [2], where $x(n)$ is lowpass filtered by a halfband filter of the form $H_{l}(z)=\frac{1}{2}\left[1+z^{-1} A\left(z^{2}\right)\right]$ which are widely used in classical PR filter bank design [9]. With the use of the so-called "noble identity" [9], the lowpass filtering operation can be carried out after downsampling as shown in Figure2. For example, if $H_{l}(z)=0.25 z+$ $0.5+0.25 z^{-1}$, then the polynomial, $A(z)=\left(1+z^{-1}\right) / 2$. In the filterbank structure of Figure 2, the subsignal $x_{2}(n)$ is adaptively predicted using the fast least squares algorithm from $x_{l}(n)$ which is a lowpass filtered and downsampled version of $x_{1}(n)$.

The above adaptive PRFB structures are extended to two dimensions in a separable manner.

\section{ADAPTIVE DENOISING}

In this section we present our image denoising algorithm which is based on the strategy presented in [5]. The corrupted image $X$ first goes through a pyramid like structure in which the lowpass filtered version of the image is subtracted from the original, and in this way the high frequency component $X_{H}$ is obtained. The image $X_{H}$ is decomposed by a filterbank and the resulting subimages are thresholded. In other words denoising is performed on $X_{H}$ instead of the corrupted image $X$. After $X_{H}$ is denoised it is added to the lowpass filtered image to obtain the restored image. In this paper, a lowpass filter with a cutoff at $\pi / 8$ is used.

In our denoising method, the adaptation strategy of the filterbank presented in Section II is modified to accommodate the edges which may cause some stability problems during the adaptation process. If an edge is detected in the prediction filter window then the LMS adaptation is stoped and a fixed filterbank is used. Once the edge is over then the LMS adaptation is started again. In this way, the convergence problem of the LMS algorithm is eliminated.

If a multistage filterbank is used in image decomposition then in the first one or two decomposition levels the adaptive filterbank is used, and in higher levels a regular fixed filterbank is used. In higher levels the actual distance between the neighboring pixels increases, and as a result adaptive prediction is not as effective as lower decomposition levels.

\section{SIMULATION STUDIES}

Our aim is to compare the performance of the adaptive filterbank to fixed filterbanks. Therefore we simply performed a single stage decomposition of the corrupted test images and obtained four subimages. Thresholding is performed on these images.

Consider the image shown in Figure 3. This image is corrupted by a zero mean Gaussian noise variance $=10$. The Mean Square Error (MSE) of this image is 100.4. Corrupted image shown in Figure 4 is analyzed by both a fixed Daubechies filter bank and the adaptive filterbank described in Section II. After regular and adaptive denoising the restored images are shown in Figures 5 and 6 respectively. The mean square errors are 30.9 and 25.6, respectively. In both cases the thresholds are experimentally determined to provide the lowest possible MSEs. 


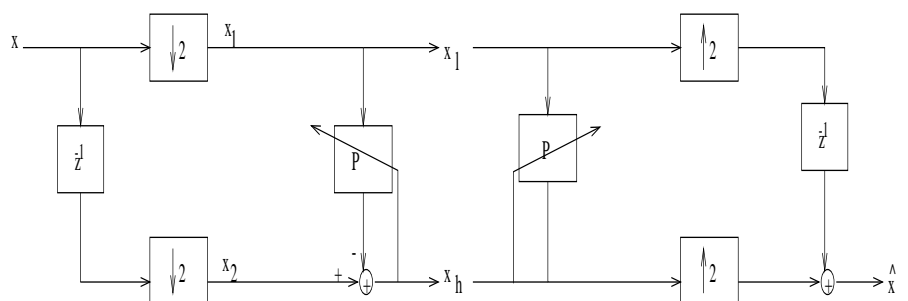

Figure 1: Analysis and synthesis stages of the 2-channel adaptive filter bank structure.

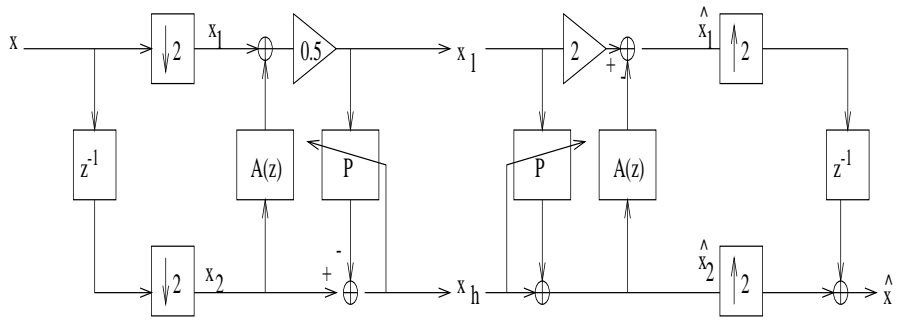

Figure 2: Adaptive filter bank structure with an antialiasing filter.

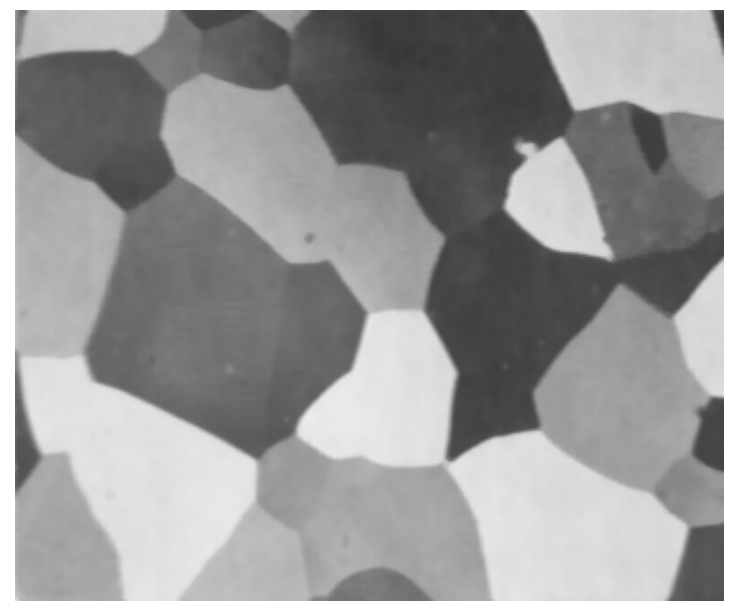

Figure 3: The original Image.

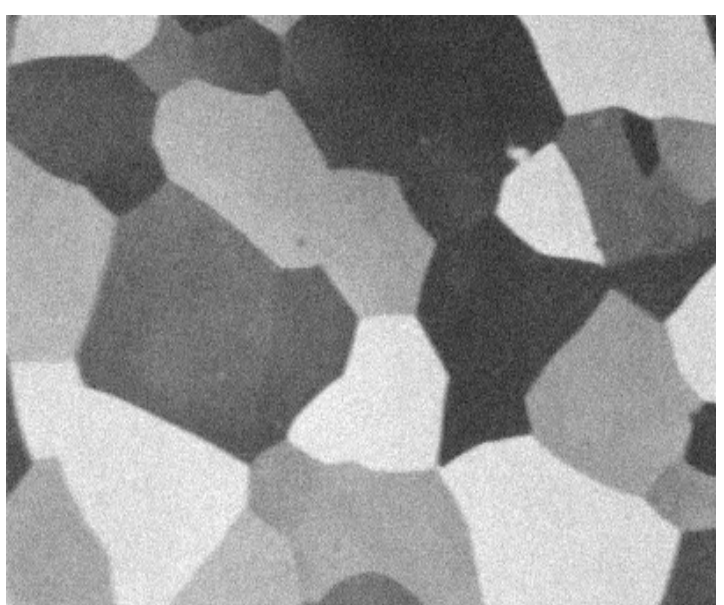

Figure 4: The Corrupted Image (MSE = 100.4).

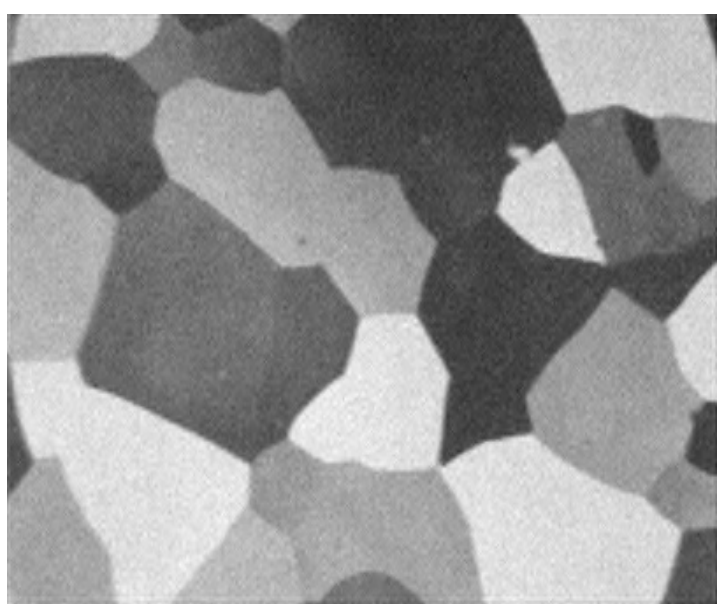

Figure 5: Restored image using a fixed filterbank (MSE = 30.9)

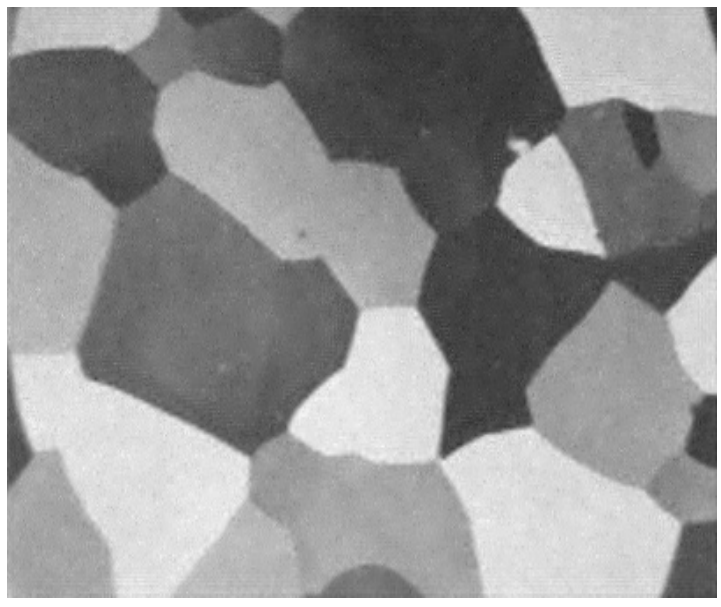

Figure 6: Restored image using the Adaptive Subband Decomposition (or Adaptive Wavelet Transform) (MSE = 25.6) 
Restored images are also evaluated by both electrical engineering students and ordinary people. In this case, 24 out of 25 people prefer the image obtained by the adaptive filterbank.

Other image restoration examples with various noise variance levels can be found in

http://www.ee.bilkent.edu.tr/ cetin/addenois.html. For example, in Image-2 (-3) [-4], \{-5\} 25 (22) [21] \{22\} out of 25 people prefer the image restored by the adaptive filterbank.

In all the test images the adaptive filterbank not only achieves lower MSE's compared to the fixed filterbanks but also the adaptively restored images are visually better than the images restored by fixed filterbanks.

\section{CONCLUSIONS}

The main contribution of this paper is the use of adaptive filterbanks instead of fixed filterbanks in denoising.

By replacing the fixed filterbanks of various denoising schemes (e.g. [5],[16, 17]) with adaptive filterbanks better image denoising results can be achieved.

Recently, it has been shown that redundant image representations are superior to orthogonal or biorthogonal filterbanks for denoising purpose. Adaptive filterbank structures described in Section II can be also extended to overcomplete structures and adaptive thresholding described in [18, 16] can be also utilized in subimages obtained using adaptive overcomplete decompositions.

\section{REFERENCES}

[1] Ö. N. Gerek, A. E. Çetin, "Linear/Nonlinear Adaptive Polyphase Subband Decomposition Structures for Image Compression," ICASSP 98, Seattle, WA, vol. 3, pp. 1345-1348, May 12-15, 1998.

[2] Ö. N. Gerek, A. E. Çetin, "Adaptive Polyphase Subband Decomposition Structures for Image Compression," IEEE Trans. on Image Processing, October 2000 .

[3] Ruşen Öktem, Enis Çetin, Levent Öktem, Karen Egiazarian, "Lossless Image Compression By LMS Adaptive Filter Banks," Signal Processing, accepted for publication in August 2000.

[4] D. Donoho, I. Johnstone, "Ideal spatial adaptation via wavelet shrinkage," Biometrika, vol. 81, pp. 425-455, 1994.
[5] J. Rosiles, Mark J. Smith, "Image Denoising Using Directional Filterbanks," Proc. IEEE ICIP-2000, Sept. 2000.

[6] O. Egger, W. Li, and M. Kunt, "High Compression Image Coding Using an Adaptive Morphological Subband Decomposition," Proc IEEE, vol. 83, no. 2, pp.272-287, February 1995.

[7] F. J. Hampson and J. C. Pesquet, "A nonlinear subband decomposition structure with perfect reconstruction," IEEE Int. Conf. on Image Proc. 1996.

[8] W. Sweldens. "The Lifting Scheme: A New Philosophy in Biorthogonal Wavelet Constructions", in Proc. of SPIE, vol. 2569, pp. 68-79, Sept. 1995.

[9] G. Strang, Wavelets and Filter Banks, Wellesley Cambridge Press, Wellesley, MA, 1996.

[10] S. Weiss, M. Harteneck, and R. W. Stewart, "On implementation and design of filter banks for subband adaptive systems," Proc. IEEE Workshop on Signal Processing Systems (SiPS'98), pp.172-181, Cambridge, MA, October 1998.

[11] N. Erdöl, F. Başbuğ, "Performance of wavelet transform based adaptive filters," Proc. IEEE ICASSP, vol. III, pp. 500-503, Minneapolis, April 1993.

[12] S. Attallah, M. Najim, "On the convergence enhancement of the wavelet transform based LMS," IEEE ICASSP, pp. 973-976, Detroit, Michigan, May 1995

[13] N. Himayat and S. A. Kassam, "A Structure for Adaptive Order Statistic Filtering,", IEEE Transactions on Image Processing, Vol. 3, pp.265-280, May 1994.

[14] Ahmed H. Tewfik, Deepen Sinha, and Paul Jorgensen, "On the Optimal Choice of a Wavelet for Signal Representation," IEEE Transactions on Information Theory, Vol. 38, No. 2, pp.747-765, Marc h 1992.

[15] Ronald R. Coifman and Mladen Victor Wickerhauser, "Entropy-Based Algorithms for Best Basis Selection," IEEE Transactions on Information Theory, Vol. 38, No. 2, pp.713-718, March 1992.

[16] M. Mihcak et.al., "Local statistical modeling of wavelet image coefficients and its application to denoising," Proc. of ICASSP-99, March 1999.

[17] J. Liu and P. Moulin, "Image denoising based on scalespace mixture modelling of wavelet coefficients," Proc. of ICIP-99, Kobe, Oct. 1999.

[18] S. G. Chang and M. Vetterli, "Spatially adaptive wavelet thresholding for image denoising," Proc. of ICIP-97, 1997. 